\title{
What can we expect from precision asteroseismology?
}

\author{
G. Handler \\ Nicolaus Copernicus Astronomical Center, Bartycka 18, 00-716 Warsaw, Poland \\ email: gerald@camk.edu.pl
}

\begin{abstract}
Precision asteroseismology is the determination of accurate stellar parameters from oscillation data. At first successful for pulsating white dwarf stars, it is now applied to more and more types of stars. We give a number of selected examples where precision asteroseismology, but also asteroseismology based on few observables may lead to considerable improvement of stellar astrophysics in the near future.
\end{abstract}

Keywords. stars: abundances, stars: atmospheres, stars: early-type, stars: fundamental parameters, stars: interiors, stars: late-type, stars: oscillations, convection, diffusion, atomic data

\section{Introduction}

The term "precision asteroseismology" was coined by Steve Kawaler and Paul Bradley in the early 1990s, when they found considerable precision in their model fits to the oscillation spectra of pulsating DO and DB white dwarf stars (e.g., Kawaler \& Bradley 1994). This work may be the first that yielded global stellar properties (for, obviously, a star other than the Sun) from the analysis of oscillation data only. Note that precise asteroseismology does not necessarily mean accurate asteroseismology, but when being able to match large numbers of observed oscillation frequencies unambiguously, there must be something fundamentally correct about the models used.

The range of topics to be covered in a contribution with the present title is wide. Hence, a rigorous selection of topics needs to be made. I have decided to restrict myself to a personally biased selection of problems related to stellar physics that may be solved in the near future thanks to asteroseismology. Other highly interesting topics, such as the study of pulsating stars as an end in itself, or what can be learned about companions to pulsating stars, or stellar aggregates that harbour them, remain ignored here.

\section{Precision asteroseismology: selected applications}

\subsection{Internal rotation}

A recent major breakthrough in the seismic study of stochastically excited pulsators concerned the determination of the interior rotation rates of some 300 red-giant stars (Mosser et al. 2012) in combination with the identification of their basic interior structure (inert or nuclear burning He-core, cf. Bedding et al. 2011). It turned out that the transition between these two phases coincides with rapid braking of core rotation, larger than the expansion of the core only would cause, and consistent with white dwarf star rotation rates. Theoretical investigations in this direction (Marques et al. 2013) suggest that rotationally induced angular momentum transfer is not efficient enough to explain this rotational braking. Efforts to obtain better resolved rotation profiles and to extend the sample of investigated stars are underway (e.g., Deheuvels et al., in preparation). The 
analysis of year-long observations of solar-like oscillators is also expected to facilitate the study of differential interior rotation of main sequence stars (Metcalfe et al. 2012).

However, it is not only the stochastically driven oscillators that can be examined this way. There is such a potential for any type of pulsator that has modes sampling the deep interior and excited to observable amplitude. For instance, the existence of differential interior rotation has been proven for some $\beta$ Cephei stars (e.g., Aerts et al. 2003, Pamyatnykh et al. 2004). It is also present in pulsating white dwarf stars (Kawaler et al. 1999), and model computations imply the possibility of rapid differential interior rotation in sdB pulsators (Kawaler \& Hostler 2005). Whereas it is clear that it will not be possible to investigate a comparably large number of $\kappa$-driven pulsators for differential rotation, it is hoped that a consistent and accurate picture of angular momentum evolution over a large part of the stellar life cycle will be obtained in the near future.

\subsection{Convective cores}

For stars more massive than the Sun, the size of their convective cores is the most important parameter that determines their main sequence lifetimes. There have been several measurements of convective core sizes for $\beta$ Cephei stars, expressed through the overshooting parameter $\alpha_{\mathrm{ov}}$. However, these do not yet result in a consistent picture (cf., e.g., Pamyatnykh et al. (2004): $\alpha_{\mathrm{ov}}<0.12$ for $\nu$ Eri and Briquet et al. (2007): $\alpha_{\mathrm{ov}}=0.44 \pm 0.07$ for $\theta \mathrm{Oph}$ ). Whereas it is not expected that $\alpha_{\mathrm{ov}}$ is constant all over the H-R diagram, it should at least be similar for stars of similar mass and evolutionary state. Perhaps we deal with precise as opposed to accurate asteroseismology here.

A first direct detection of a convective core in a stochastically excited main sequence pulsator has recently been made (Silva Aguirre et al. 2013). It was found to extend beyond the Schwarzschild convective boundary, which could be a hint towards the presence of overshooting. However, the imprints of a convective core present in the main sequence stage of evolution can still be found in the oscillation spectra of subgiant stars (Deheuvels \& Michel 2011), yielding another possibility to determine convective core sizes.

\subsection{Opacities}

An example of the differences between precise and accurate asteroseismology can be found in Daszyńska-Daszkiewicz \& Walczak (2010): fitting $\beta$ Cep star pulsation spectra with different opacity tables will yield different global parameters. More importantly, these authors showed (Daszyńska-Daszkiewicz \& Walczak 2009) that the observed ratio of the bolometric flux to radius variation in hot pulsating stars can be used to constrain which opacities better reproduce the observations - and the results for the stellar interior may be different than for the surface regions. This topic is discussed in detail by Walczak (these proceedings).

\section{Precise asteroseismology from few observables}

It does not always require to fit large numbers of pulsation modes to obtain important asteroseismic results. This does of course not mean that this type of asteroseismology is not precise, this separation is rather a question of concept.

\subsection{The $\epsilon$ mechanism}

Pulsational driving via variations in nuclear energy generation has historically often been suspected to be a feasible mechanism (e.g., see Kawaler 1988), but observational confirmation has never been attained. Recently, this topic has reappeared in several different contexts. For instance, the long pulsation periods of a He-rich subdwarf B star 
could only be reproduced with an $\epsilon$ mechanism operating in unstable He-burning shells. It has also been suggested that a small fraction of the remnants of double He-white dwarf mergers should show such oscillations (Miller Bertolami et al. 2013).

In an attempt to explain the variability of Rigel, Moravveji et al. (2012) found that the $\epsilon$ mechanism in the H-burning shell would be able to excite high-order g-mode pulsations in blue supergiants. If so, asteroseismology of immediate core-collapse supernova progenitors would become possible.

The $\epsilon$ mechanism may also be responsible for low-order g-mode excitation in some GW Vir stars (see Córsico et al. 2009), but an observational detection has not yet convincingly been made. Other recent theoretical investigations showing the possibility of pulsations excited by the $\epsilon$ mechanism comprise Sonoi \& Shibahashi (2009) for models of very low metallicity in the domain of the $\gamma$ Dor stars, and Rodríguez-López et al. (2012) for models of $\mathrm{M}$ dwarfs.

\subsection{Further sources of excitation}

The rapidly oscillating Ap stars are believed to be excited by the $\kappa$ mechanism in the $\mathrm{H} / \mathrm{He}$ I ionization zone. However, theoretical calculations of their instability strip (Cunha 2002) do not match the observed locations of the stars well. In addition, a few stars show pulsations with frequencies higher than the acoustic cut-off frequency.

Similarly, some $\delta$ Scuti stars have very large ranges of oscillation frequencies excited that are too wide to be driven only by the $\kappa$ mechanism in the He II ionization zone (e.g., Antoci et al. 2011). These problems may be resolvable if the turbulent pressure in the near-surface convection zone acted as an additional driving agent (e.g., see Antoci, these proceedings).

Atomic diffusion may also add to pulsational driving (e.g. in main sequence B stars, sdB pulsators or $\gamma$ Dor stars) by producing suitable accumulation of elements to enhance the $\kappa$ effect (e.g., see Théado et al. 2009). A new opacity bump has recently been identified (Cugier 2012) from improved model atmosphere data and its effects on stellar pulsation spectra are being investigated (Cugier, these proceedings).

\subsection{Surface convection}

Three-dimensional simulations (Trampedach \& Stein 2011) allow to predict the mixinglength parameter of surface convection in the cooler part of the H-R diagram. These results can be tested by model calculations based on the large frequency separation and frequency of maximum power observed in stochastically excited pulsators (Bonaca et al. 2012). From the latter study it is evident that there are still problems. First, the rather strong dependence of the mixing-length parameter on temperature predicted by the models could not yet be confirmed. Additionally, using the solar value for the mixing length instead, a problem with the resulting helium abundance arises: it is in many cases below the primordial one. Whatever the solution to this dilemma, a better understanding of surface convection or other physics of solar-like stars is to be expected.

\section{Acknowledgements}

I am grateful to the scientific organizers of this meeting for entrusting me with this presentation. My attendance of the conference has been supported by the Polish NCN grant 2011/01/B/ST9/05448.

\section{References}

Aerts, C., Thoul, A., Daszyńska, J., et al. 2003, Science, 300, 1926 
Antoci, V., Handler, G., Campante, T., et al. 2011, Nature, 477, 570

Bedding, T. R., Mosser, B., Huber, D., et al. 2011, Nature, 471, 608

Bonaca, A., Tanner, J. D., Basu, S., et al. 2012, ApJ, 755, L12

Briquet, M., Morel, T., Thoul, A., et al. 2007, MNRAS, 381, 1482

Córsico, A. H., Althaus, L. G., Miller Bertolami, M. M., González Pérez, J. M., \& Kepler, S. O. 2009, ApJ, 701, 1008

Cugier, H. 2012, A\& $A, 547, \mathrm{~A} 42$

Cunha, M. S. 2002, MNRAS, 333, 47

Daszyńska-Daszkiewicz, J. \& Walczak, P. 2009, MNRAS, 398, 1961

Daszyńska-Daszkiewicz, J. \& Walczak, P. 2010, MNRAS, 403, 496

Deheuvels, S. \& Michel, E. 2011, A\&A, 535, A91

Kawaler, S. D. 1988, ApJ, 334, 220

Kawaler, S. D. \& Bradley, P. A. 1994, ApJ, 427, 415

Kawaler, S. D. \& Hostler, S. R. 2005, ApJ, 621, 432

Kawaler, S. D., Sekii, T., \& Gough, D. O. 1999, ApJ, 516, 349

Marques, J. P., Goupil, M. J., Lebreton, Y., et al. 2013, A\&A, 549, A74

Metcalfe, T. S., Chaplin, W. J., Appourchaux, T., et al. 2012, ApJ, 748, L10

Miller Bertolami, M. M., Córsico, A. H., Zhang, X., Althaus, L. G., \& Jeffery, C. S. 2013, in: J. Montalbán, A. Noels, \& V. Van Grootel (eds.) Ageing Low Mass Stars: From Red Giants to White Dwarfs, EPJ Web of Conferences, Vol. 43, id. 04004

Moravveji, E., Moya, A., \& Guinan, E. F. 2012, ApJ, 749, 74

Mosser, B., Goupil, M. J., Belkacem, K., et al. 2012, A $₫ A$, 548, A10

Pamyatnykh, A. A., Handler, G., \& Dziembowski, W. A. 2004, MNRAS, 350, 1022

Rodríguez-López, C. \& MacDonald, J., Moya A. 2012, MNRAS, 419, L44

Silva Aguirre, V., Basu, S., Brandão, I. M., et al. 2013, ApJ, 769, 141

Sonoi, T. \& Shibahashi, H. 2012, MNRAS, 422, 2642

Théado, S., Vauclair, S., Alecian, G., \& LeBlanc, F. 2009, ApJ, 704, 1262

Trampedach, R. \& Stein, R. F. 2011, ApJ, 731, 78 